\title{
Minimally displaced acetabulum fractures in geriatric patients: a comparison of open, percutaneous and non-operative treatment from the German Pelvic Injury Register data
}

\author{
Helene Ernstberger ${ }^{1} \cdot$ Philipp Pieroh $^{1} \cdot$ Andreas Höch $^{1} \cdot$ Christoph Josten $^{1} \cdot$ Steven C. Herath ${ }^{2} \cdot$ Georg Osterhoff $^{1}$. \\ Working Group on Pelvic Fractures of the German Trauma Society
}

Received: 18 December 2019 / Accepted: 10 March 2020 / Published online: 8 April 2020

(c) The Author(s) 2020

\begin{abstract}
Purpose In elderly patients with minimally displaced acetabulum fractures, the patients' inability to partially weight-bear and the need for early mobilisation may trigger the decision towards a treatment with higher primary stability. The purpose of this study was to compare open reduction and internal fixation (ORIF), closed reduction and percutaneous fixation (CRPIF) and non-operative treatment in geriatric minimally displaced acetabulum fractures with regard to complications and quality of reduction.

Methods Data from the prospective German Pelvic Injury Register collected between 2008 and 2018 were used to evaluate 608 geriatric patients with isolated minimally displaced $(\leq 5 \mathrm{~mm})$ acetabulum fractures. In total, 429 received non-operative treatment, 117 ORIF and 62 CRPIF. Demographics, injury severity, fracture pattern, complications and fracture displacement before and after treatment were analysed.

Results Both operative methods reduced fracture gap displacement. CRPIF was associated with lower blood loss and shorter operative time compared to ORIF ( $p<0.001)$. Hospital stay was 12.9 days in the non-operative group, 16.8 with CRPIF and 23.6 with ORIF $(p<0.001)$. Non-surgical general complications were more likely to occur following ORIF $(22.2 \%)$ compared to CRPIF $(8.1 \%)$ and non-operative treatment $(8.4 \%, p<0.001)$. The rate of surgical complications was not different for ORIF and CRPIF $(p=0.122)$

Conclusion Both operative treatments improve fracture displacement and joint congruency in elderly patients with minimally displaced acetabulum fractures. Compared to ORIF, CRPIF achieves similar quality of reduction but is associated with fewer complications, smaller intraoperative blood loss, shorter operative time and shorter length of hospital stay.
\end{abstract}

Keywords Acetabulum fracture $\cdot$ Elderly $\cdot$ Percutaneous fixation $\cdot$ ORIF $\cdot$ Conservative treatment $\cdot$ Reduction

\section{Introduction}

The incidence of acetabular fractures in the elderly has increased by 2.4 -fold since 1980 [1] and represents the most rapidly growing group within acetabular injuries [2]. Many studies have outlined the differences of acetabulum fractures

Helene Ernstberger

Helene.Ernstberger@medizin.uni-leipzig.de

1 Department of Orthopaedics, Trauma and Plastic Surgery, University Hospital Leipzig, 04103 Leipzig, Germany

2 Department of Trauma, Hand and Reconstructive Surgery, Saarland University Hospital, Building 57, Kirrbergerstr. 1, 66421 Homburg, Germany between young and old ages $[1,3]$. The mechanism of injury in young patients is mainly high-energy trauma, more frequently affecting the posterior parts of the acetabulum. In the geriatric group, the trauma is most commonly a simple fall and more likely to be associated with anterior column fractures [1,3]. Consequently, different standards of treatment should apply for the elderly than those used in young ages.

Treatment options include conservative treatment, open reduction and internal fixation (ORIF) and closed reduction and percutaneous fixation (CRPIF). Minimally displaced acetabular fractures usually can be considered stable and thus be treated non-operatively [3, 4]. A recent study even showed good functional outcome for acetabulum fractures with greater displacement and non-operative treatment in 
the elderly [5]. However, as prolonged bed rest is associated with increased morbidity and mortality [6], early mobilisation is essential [7]. ORIF allows for direct visualization of the fragments and has the potential to result in a good quality of reduction and early mobilisation [3]. In young patients, better reduction is associated with a better clinical outcome and a lower rate of secondary osteoarthritis [3, 8-12]. Many studies identified higher age as a negative predictor regarding reduction, mortality and complications, though [1, 13-15]. Percutaneous screw fixation of acetabulum fractures has the advantage of less invasive approaches and lower blood loss and, hence, seems to be a potential alternative for geriatric patients [15-17]. However, percutaneous techniques only allow for closed reduction and may result in inferior fracture reduction when compared to ORIF. Which treatment is best for elderly patients is still under debate.

The aim of this study was to compare open operative, percutaneous operative and non-operative treatment of acetabulum fractures in geriatric patients with regard to nonsurgical general and surgical complications, intraoperative blood loss, and quality of reduction achieved.

\section{Materials and methods}

A retrospective multicentre registry study was conducted analysing the register of the German pelvic work group (Pelvic Injury Register of the German Trauma Society), as part of the German Society of Traumatology (DGU) and German section of the AO Trauma.

This study was approved by the local institutional ethics committee (Ethik-Kommission Leipzig, 119/19-ek).

\section{Patients}

In total, 3432 patients with acetabulum fractures were documented in the German Pelvic Trauma Registry from 07/2008 until 03/2018. Patients aged 60 years and older with a preoperative gap/step of $\leq 5 \mathrm{~mm}$ were included. Within the inclusion criteria, 829 patients were eligible.

Patients with combined acetabulum and pelvic ring fractures of type B/C (tile classification $[18,19]$ ) or sacral fractures $(n=156)$, with bilateral acetabular fractures $(n=16)$, periprosthetic fractures $(n=33)$ fractures due to metastatic disease $(n=4)$ and those treated with primary total hip arthroplasty $(n=10)$ were excluded. In addition, we had to exclude two patients receiving THA half a year after the injury, as information about initial treatment for the fracture was missing. Finally, 608 patients were analysed for this study. Patients were assigned to three groups: open reduction and internal fixation (ORIF, $n=117)$, closed reduction and percutaneous internal fixation (CRPIF, $n=62$ ), and non-operative treatment $(n=429)$. For further analysation, patients were separated in two groups: 60-79-year-old $(n=349)$ and $80-100$-year-old $(n=259)$ patients.

\section{Data acquisition}

All data were extracted from the central database of the Pelvic Injury Register of the German Trauma Society. For statistical analyses, data were selected and transferred into a SPSS table. This information included age and gender, fracture pattern according to Judet and Letournel [3], hospital duration and the treatment method. Based on plain radiography (anteroposterior pelvic view), the fracture gap and step had been evaluated before and after surgery. Complications that could be directly linked to the surgical procedure were documented as "surgical complications". Complications that occurred during the hospitalisation but without direct reference to the surgical procedure were documented as "nonsurgical general complications". For the operative treatment groups, operation duration and intraoperative blood loss were documented. Patients were followed up until their day of discharge.

\section{Statistical analysis}

Statistics were performed using SPSS 24.0 (SPSS Inc., Chicago, IL, USA). Continuous data are presented as mean with standard deviation (SD), categorical data as absolute number of cases $(n)$ and percentage (\%).

Primary outcome was the occurrence of general complications during hospital stay and surgical complications. A Pearson Chi-square test was used to assess differences regarding complication rates between the three groups and between 60-79 and 80-100-year-old patients. Likewise, a Chi-square test was performed to compare the two surgical methods concerning surgical complications. As secondary outcome, we analysed the hospital duration, quality of reduction, intraoperative blood loss and operative time. Metric, non-Gaussian variables, in our case age, ISS score, hospital duration and fracture gap/step were evaluated performing a Kruskal-Wallis $H$ test. Concerning paired comparison, post hoc tests with Dunn-Bonferroni correction were executed. A Mann-Whitney $U$ test was chosen to detect differences regarding intraoperative blood loss, since the variable exhibited non-normal distribution, and fracture gap/step regarding age. As for 12 patients treated openly, a blood loss of $0 \mathrm{ml}$ was documented, these patients had to be excluded analysing this variable. The operative time was evaluated using a Student's $T$ test. A $p$ value of $<0.05$ was considered as statistically significant. Boxplots were prepared to illustrate changes of fracture gap/step using operative treatment and in comparison to non-operative treatment. 


\section{Results}

\section{Patients' baseline characteristics}

The mean age at time of the trauma was 77.3 (SD 9.7) years (range 60-100), 227 (37.3\%) were of female gender (Table 1). The mean injury severity score (ISS) was 10.9 (SD 5.79), showing similar scores in all three groups $(p=0.546)$.

Patients receiving non-operative treatment were significantly older than patients treated by CRPIF $(p=0.004)$ or ORIF $(p<0.001)$, whereas patients who were treated by CRPIF or ORIF were not different in age $(p=1.00)$. The most common fracture pattern according to Judet and Letournel [3] was an isolated anterior column fracture $(n=191 ; 31.4 \%)$, followed by combined anterior column posterior hemitransverse $(n=123 ; 20.2 \%)$ and anterior wall fractures $(n=94 ; 15.5 \%$, Table 2$)$.

\section{Complications}

Non-surgical complications were documented for 67 (11.0\%, Table 3) patients. There was no significant difference between patients treated by CRPIF $(n=5 ; 8.1 \%)$ and non-operatively treated patients $(n=36 ; 8.4 \%, p=0.931)$. With ORIF, however, $22.2 \%(n=26)$ showed complications, which was a significantly higher rate compared to the other groups (CRPIF: $p=0.017$; non-op.: $p<0.001$ ). Concerning all patients, the most common complication documented was haematoma $(n=10)$. There were two patients with thrombosis, five with pulmonary embolism, three with acute respiratory distress syndrome (ARDS), two with multi-organ failure, seven neurologic complications, four superficial and seven deep wound infections, four bleedings, one secondary displacement and one case with delayed wound healing. For 36 patients with different complications from those mentioned above, "other non-surgical complications" were documented, summarised in Table 4. As three patients had more than one "other non-surgical complication" there are
Table 1 Patients' baseline characteristics

Table 2 Fracture patterns

\begin{tabular}{llllll}
\hline & \multicolumn{1}{l}{ Treatment } & & & \\
\cline { 2 - 6 } & Non-operative & Percutaneous & Open & $p$ & Total \\
\hline$N$ & 429 & 62 & 117 & 608 \\
Age (years) & $78.7(9.71)$ & $74.3(10.02)$ & $74.1(8.08)$ & $0.000^{\lrcorner}$ & $77.3(9.66)$ \\
Gender (f:m) & $185: 244$ & $12: 50$ & $30: 87$ & & $227: 381$ \\
Fracture gap after trauma (mm) & $1.59(1.26)$ & $2.47(1.46)$ & $3.11(1.57)$ & $0.000^{\lrcorner}$ & $1.97(1.48)$ \\
Fracture step after trauma (mm) & $0.97(1.20)$ & $1.32(1.38)$ & $2.37(1.54)$ & $0.000^{\lrcorner}$ & $1.28(1.40)$ \\
\hline
\end{tabular}

${ }^{\lrcorner}$Kruskal-Wallis $H$ test, SD in brackets

\begin{tabular}{lllll}
\hline & \multicolumn{3}{l}{ Treatment } & \\
\cline { 2 - 5 } & Non-operative & Percutaneous & Open & Total \\
\hline $\mathrm{N}$ & 429 & 62 & 117 & 608 \\
Fracture pattern & & & & \\
$\mathrm{PW}$ & $32(7.5 \%)$ & 0 & $8(6.8 \%)$ & $40(6.6 \%)$ \\
$\mathrm{PC}$ & $20(4.7 \%)$ & $2(3.2 \%)$ & $4(3.4 \%)$ & $26(4.3 \%)$ \\
$\mathrm{AW}$ & $88(20.5 \%)$ & $3(4.8 \%)$ & $3(2.6 \%)$ & $94(15.5 \%)$ \\
$\mathrm{AC}$ & $141(32.9 \%)$ & $30(48.4 \%)$ & $20(17.1 \%)$ & $191(31.4 \%)$ \\
Transverse & $23(5.4 \%)$ & $4(6.5 \%)$ & $2(1.7 \%)$ & $29(4.9 \%)$ \\
PW/PC & $1(0.2 \%)$ & 0 & $3(2.6 \%)$ & $4(0.7 \%)$ \\
Transverse/PW & $4(0.9 \%)$ & $1(1.6 \%)$ & $7(6.0 \%)$ & $12(2.0 \%)$ \\
T shaped & $27(6.3 \%)$ & $2(3.2 \%)$ & $4(3.4 \%)$ & $33(5.4 \%)$ \\
ACPHT & $71(16.6 \%)$ & $15(24.2 \%)$ & $37(31.6 \%)$ & $123(20.2 \%)$ \\
Both columns & $15(3.5 \%)$ & $4(6.5 \%)$ & $28(23.9 \%)$ & $47(7.7 \%)$ \\
Unknown & $2(0.5 \%)$ & 0 & 0 & $2(0.3 \%)$ \\
No classification & $5(1.2 \%)$ & $1(1.6 \%)$ & $1(0.8 \%)$ & $7(1.2 \%)$ \\
\hline
\end{tabular}

$P W$ posterior wall, $P C$ posterior column, $A W$ anterior wall, $A C$ anterior column, $A C P H T$ anterior column posterior hemitransverse 
Table 3 Outcome

\begin{tabular}{llllll}
\hline \multicolumn{5}{l}{ Treatment } \\
\cline { 2 - 6 } & Non-operative & Percutaneous & Open & $p$ & Total \\
\hline$N$ & 429 & 62 & 117 & & 608 \\
OR duration (min) & - & $91(52)$ & $169(67)$ & $0.000^{\dagger}$ & $142(73)$ \\
Blood loss (ml) & - & $73(184)$ & $639(451)$ & $0.000^{\ddagger}$ & $429(464)$ \\
Non-surgical complications & $36(8.4 \%)$ & $5(8.1 \%)$ & $26(22.2 \%)$ & $0.000^{*}$ & $67(11.0 \%)$ \\
Surgical complications & - & $3(4.8 \%)$ & $14(12.0 \%)$ & $0.122^{*}$ & $17(9.5 \%)$ \\
Hospital duration (days) & $12.9(12.8)$ & $16.8(12.0)$ & $23.6(15.2)$ & $0.000^{\lrcorner}$ & $15.3(13.8)$ \\
Fracture gap after treatment $(\mathrm{mm})$ & - & $1.2(1.3)$ & $1.3(1.0)$ & $1.00^{\lrcorner}$ & $1.2(1.1)$ \\
Fracture step after treatment $(\mathrm{mm})$ & - & $0.5(1.0)$ & $0.8(1.0)$ & $0.844^{\lrcorner}$ & $0.7(1.0)$ \\
\hline
\end{tabular}

$\times 12$ patients in the ORIF group had to be excluded due to blood loss of $0 \mathrm{ml}$

$O R$ operation room

*Pearson Chi-square test

${ }^{\dagger}$ Student's $T$ test

${ }^{\ddagger}$ Mann-Whitney $U$ test

${ }^{\lrcorner}$Kruskal-Wallis H test, SD and percentage in brackets

36 patients, but 42 complications listed in Table 4 . From the registry data available, it was not obvious what caused the infections, bleedings, drop of haemoglobin and anaemia and whether they occurred due to surgery or other injuries. Therefore, they were assigned to "non-surgical general complications". Infections and bleedings with known relation to the surgical procedure were documented as "surgical complications". Surgical complications included deep infection $(n=4)$, intraoperative bleeding $(n=2)$, implantat malpositioning or loosening with $(n=3)$ and without $(n=2)$ re-osteosynthesis and six "other complications" (Table 4). Surgical complications occurred in 3/62 cases with CRPIF (4.8\%) compared to ORIF $(14 / 117,12.0 \%)$ but the difference was not significant $(p=0.122)$. In total, 17 patients $(9.5 \%)$ had surgical complications (Table 3 ).

Comparing 60-79-year-old patients with 80-100-yearold patients, there was no significant difference regarding non-surgical complications (60-79: 38 (10.9\%); 80-100: 29 $(11.2 \%) ; p=0.904)$ and surgical complications (60-79: 11 (8.3\%); 80-100: 6 (8.7\%); $p=0.384)$. However, after receiving CRIPF, no patient aged 60-79 had a surgical complication $(0 / 43$ cases; $0 \%)$ but 3 out of 19 patients aged $80-100$ $(15.8 \%)(p=0.026)$.

\section{Length of operation and hospitalisation, and intraoperative blood loss}

The mean hospital stay length was 15.3 days (SD 13.8, Table 3). Non-operative treatment was associated with the shortest mean hospital stay, followed by CRPIF $(p<0.001)$ and ORIF $(p<0.001)$. To measure the influence of the different follow-up times, a partial correlation was being performed, showing that the complication rate after ORIF was no longer higher than after CRPIF and non-operative treatment $(p=0.758)$.

Both, the mean operation time $(p<0.001)$ and intraoperative blood loss $(p<0.001)$ were significantly lower when the surgery was performed percutaneously (Table 3 ).

\section{Quality of reduction}

Mean fracture gap after trauma was $2.0 \mathrm{~mm}$ (1.5 SD), mean fracture step $1.3 \mathrm{~mm}$ (1.4 SD). Patients treated non-operatively had a significant lower mean posttraumatic articular gap compared to patients where the decision for open $(p<0.001)$ or percutaneous $(p<0.001)$ surgery had been made (Table 1, Figs. 1, 2). The mean posttraumatic, pretreatment articular step in the non-operative group was similar to that seen in the CRPIF group $(p=0.274)$ but smaller compared to the ORIF group $(p<0.001)$.

Both operative methods achieved significant articular fracture gap and step reduction $(p<0.05$, Figs. 1, 2) when comparing postoperative to posttraumatic displacement. Concerning quality of reduction, no difference could be found between percutaneous and open treatment (gap: $p=1.00$; step: $p=0.844$ ). Percutaneous fixation reduced fracture step/gap to smaller dimensions than those seen in non-operatively treated patients (gap: $p=0.049$; step: $p=0.025$ ).

Comparing 60-79-year-old patients with 80-100-yearold patients, no significant difference can be seen regarding articular displacement in the non-operative group (gap: $p=0.068$; step: $p=0.153$ ) after ORIF (gap: $p=0.725$; step: $p=0.361$ ) and after CRPIF (gap: $p=0.083$; step: 
Table 4 Other complications

\begin{tabular}{|c|c|c|c|}
\hline & Non-operative & Percutaneous & Open \\
\hline \multicolumn{4}{|l|}{ Other non-surgical complications } \\
\hline Pulmonary oedema & & 1 & \\
\hline Atelectasis & & & 1 \\
\hline Pneumonia & 3 & 1 & 1 \\
\hline Pulmonary aggravation & 1 & & \\
\hline Haemothorax & 1 & & \\
\hline Cardiac decompensation & & & 1 \\
\hline Pacemaker due to cardiac arrythmia & & & 1 \\
\hline NSTEMI & 1 & & \\
\hline Subacute myocardial ischemia & 1 & & \\
\hline Postoperative anaemia & & & 1 \\
\hline Drop of haemoglobin & & & 1 \\
\hline Urinary tract infection & 3 & 1 & 1 \\
\hline Renal failure & 1 & & \\
\hline Renal failure of the transplanted renal & 1 & & \\
\hline Secondary necrosis of the femoral head & & & 1 \\
\hline Acute hepatitis & 1 & & \\
\hline Sub ileus & 1 & & \\
\hline Caecum perforation & & & 1 \\
\hline Pneumoperitoneum with diagnostic laparotomy & & & 1 \\
\hline Perianal bleeding & 1 & & \\
\hline MRSA skin & & & 1 \\
\hline Fall with laceration of the finger and face & 1 & & \\
\hline Decubitus & 3 & & \\
\hline Delir & 1 & & \\
\hline TIA & 1 & & \\
\hline Seizure & 1 & & \\
\hline Loss of consciousness & 1 & & \\
\hline TEP loosening of the other hip & 1 & & \\
\hline Free joint body & & & 1 \\
\hline Pain & 1 & & \\
\hline TUR prostate & 1 & & \\
\hline Carbon dioxide narcosis & 1 & & \\
\hline \multicolumn{4}{|l|}{ Other surgical complications } \\
\hline No reduction & & 1 & \\
\hline Dorsal extra osseous screw & & & 1 \\
\hline Broken intra-articular K-wire & & & 1 \\
\hline Postoperative bleeding ( 2 times) & & & 1 \\
\hline Postoperative haematoma & & & 1 \\
\hline Caecum perforation & & & 1 \\
\hline
\end{tabular}

MRSA multi-resistant Staphylococcus aureus, TIA transient ischemic attack, TEP total endoprosthesis, TUR transurethral resection

$p=0.419$ ). However, in patients aged $80-100$, fracture gap and step could not be reduced significantly using CRPIF (gap: $p=0.134$; step: $p=1.00$ ), but using ORIF (gap: $p<0.001$; step: $p<0.001$ ).

\section{Discussion}

The optimal treatment of minimally displaced acetabulum fractures in the elderly remains controversial. The 
Fig. 1 Fracture gap of minimal displaced acetabulum fractures. Comparison of fracture gap in mm of open reduction and internal fixation (ORIF) before and after surgery (red), percutaneous treatment before and after surgery (blue) and non-operative treatment (black stripes). The boxes show the percentiles 25, 50 (Median) and 75. The end of the whiskers shows $1.5 \times$ interquartile range. Outliers and extremes are not shown. A Kruskal-Wallis $H$ test with Dunn-Bonferroni correction was performed

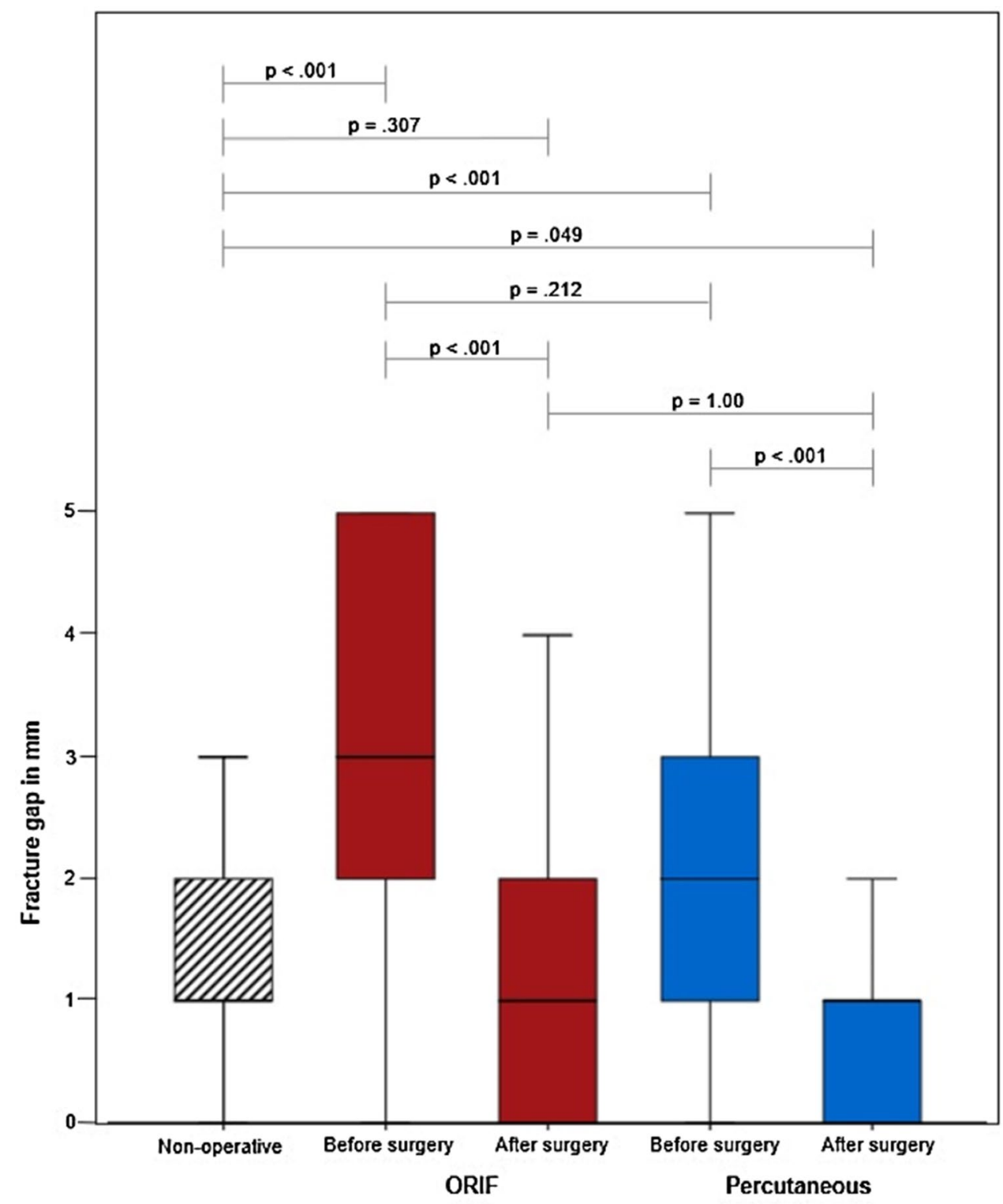

advantage of better primary stability with operative fixation has to be weighed against the potential complications of a surgical intervention. Surgery, however, can be performed in different degrees of invasiveness. This study compared ORIF, CRPIF and non-operative treatment regarding non-surgical and surgical complications, intraoperative blood loss, and quality of reduction.

The fracture patterns seen in this study were mainly those affecting the anterior column of the acetabulum. These are the common patterns described for geriatric patients and one markedly difference between old and young individuals $[1,3,20,21]$. It underlines even more the need for research aimed specifically for geriatric acetabulum fractures.

In this study, using any of the two operative methods achieved significant reduction of articular fracture displacement. Many studies have underlined optimal reduction as an important factor for good clinical outcome and prevention of late complications [4, 9-13, 22, 23]. Regardless of age, Matta et al. highlighted the importance of fracture reduction to at least 3-mm gap and step [24]. Verbeek et al. described a cut-off at 5-mm gap and 1-mm step, others mentioned a cut-off at 2-mm gap and step as a measure for a good outcome [4, 25]. The mean values that could be achieved by operative treatment in this study remain below these thresholds. This might be somewhat unexpected, since several authors seemed to expect worse outcome for percutaneous treatment [17, 26, 27]. Most of these studies are old and, as minimal invasive treatment is developing, better results can be expected now [28-30]. However, studies comparing quality of reduction are rare. To the authors' knowledge, only Jeffcoat et al. compared an open and limited ilioinguinal approach with the result 
Fig. 2 Fracture step of minimal displaced acetabulum fractures. Comparison of fracture step in $\mathrm{mm}$ of open reduction and internal fixation (ORIF) before and after surgery (red), percutaneous treatment before and after surgery (blue) and non-operative treatment (black stripes). The boxes show the percentiles 25, 50 (Median) and 75. The end of the whiskers shows $1.5 \times$ interquartile range. Outliers and extremes are not shown. A Kruskal-Wallis $H$ test with Dunn-Bonferroni correction was performed

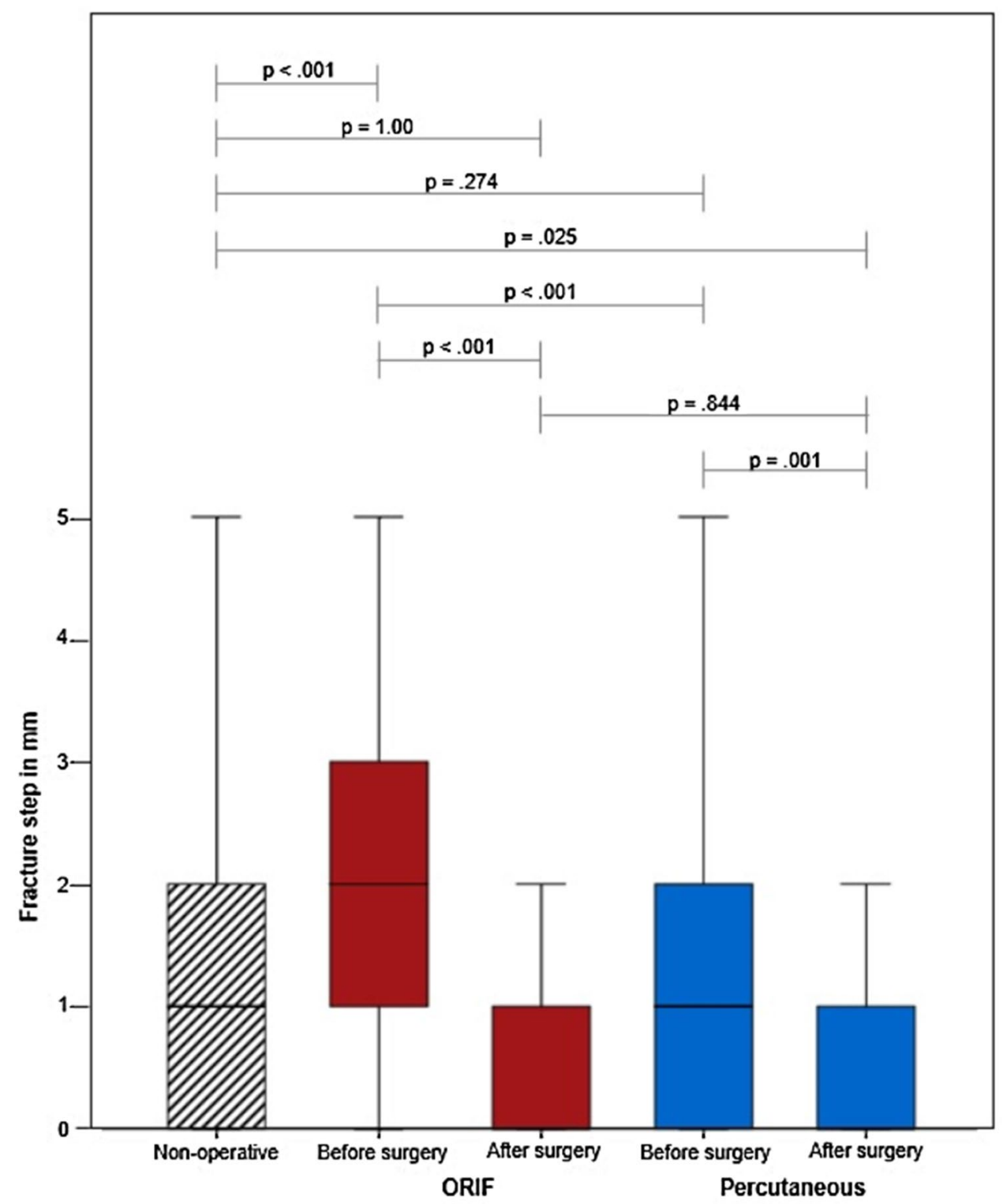

of equivalent reduction for both methods, similar to the findings of this study [28].

Fracture gap after trauma was significant smaller in the non-operative group compared with the two operative groups. The size of displacement has always been important for the decision, whether patients should be operated or can be treated conservatively $[3,4]$. Likewise, it is well accepted that residual steps are less tolerable than residual gaps [4, 25]. Already with a 5-mm gap and a 1-mm articular step [25], surgery should be considered.

From a prognostic point of view, fracture displacement of up to $5 \mathrm{~mm}$ could not be specified as minimally displaced. However, the aim of this study is to compare non-operative, ORIF and CRPIF treatment for acetabulum fractures. Several studies have shown, that highly displaced fractures (average initial displacement of $16 \mathrm{~mm}$ up to $30 \mathrm{~mm}$ ) can be reduced adequately $[23,29,31]$. Therefore, a displacement of maximum $5 \mathrm{~mm}$ only represents minimally displaced fractures. This terminology has already been used in the literature [32].

It was shown that CRPIF can reduce blood loss and operative time significantly compared to ORIF. These findings are consistent with the previous literature [15, 16, 28]. Since short operative time and low blood loss are substantial, especially for geriatric patients with low cardiac reserve [33, 34], minimal invasive treatment options represent a conceivable alternative.

The length of hospital-stay and complication rates was low in patients treated non-operatively or by CRPIF. A long hospital stay is associated with prolonged bed rest resulting in cardiovascular, respiratory, musculoskeletal and psychological complications $[6,35]$. 
Non-surgical complications occurred more likely with ORIF. The influence of the treatment method on the complication rate cannot be seen independent of hospital stay length, though. Performing a partial correlation to measure the impact of the different follow-up times, the complication rate after ORIF was no longer higher than after CRPIF and non-surgical treatment. But whether patients stayed in hospital due to their complications or complications occurred due to a prolonged hospitalisation cannot be told from the registry data available. Naturally, a more invasive surgery requires a longer monitoring of the patient.

There were no differences between open and percutaneous methods when looking at only surgical complications, however,- - as this has been described for ORIF and CRPIF in the previous literature [28].

MRSA skin was captured as "non-surgical complication" since hospital-acquired MRSA colonialization occurs during hospital stay and may lead to serious MRSA infections especially in combination with surgical wounds [36].

There are several limitations concerning this study. First, registry studies do not allow for integration of variables not documented in the registry a priori, such as comorbidities, radiographic results of non-operative treatment at day of discharge, information about the weight-bearing area, indications for surgery and conservative treatment, postoperative management, weight-bearing and functional outcome. The range of age (60-100) is wide. Separating the patient population in 60-79-year-old and 80-100-year-old patients, the subgroups got too small for adequate statistical analysation (e.g., patients $\geq 80$ years treated by CRPIF $n=19$ ). Therefore, we can only make a limited guess and further research would be needed to specify the treatment methods in the context of geriatric age. We excluded fractures exhibiting a gap/step of more than $5 \mathrm{~mm}$. Thus, our findings allow only conclusions for minimally displaced acetabulum fractures. Last, the follow-up time is limited to the hospital stay length and does not reflect late complications and the development of osteoarthritis. Differences may occur after discharge and, therefore, further studies comparing complications with long-term follow-up would be useful. It would be interesting to evaluate whether there is a difference in long-term complications depending on the residual displacement in different ages. Despite these limitations, this study presents data from a large multi-centric series on treatment of minimally displaced acetabulum fractures in the elderly.

Open reduction and internal fixation is the gold standards for young patients. In geriatric patients, the potential disadvantages of the higher invasiveness even of strictly intrapelvic or pararectus approaches must be considered.

Hence, conservative treatment has been described as a valid method in selected cases [5, 23, 37]. This study highlights a short hospital stay length and low rate of in hospital complications for non-operative treatment.
Percutaneous operative techniques, may pose a valuable alternative in geriatric patients that provides both: primary stability and minor surgical trauma. In this cohort of minimally displaced acetabulum fractures, CRPIF did not come at the cost of inferior quality of reduction. However, these results may not be generalised as radiographic outcome is not necessarily linked to functional outcome in all cases, especially in elderly patients. Individual considerations would be necessary for every patient. Minimal invasive methods are challenging and surgeons have to consider their own abilities [38]. Yet, imaging techniques are improving and knowledge is increasing, which will facilitate the application of these techniques in the future [30].

\section{Conclusion}

Both operative treatments improve fracture displacement and joint congruency in elderly patients with minimally displaced acetabulum fractures. Compared to ORIF, CRPIF achieves similar quality of reduction but is associated with fewer complications, smaller intraoperative blood loss, shorter operative time and shorter length of hospital stay.

Acknowledgements Open Access funding provided by Projekt DEAL.

\section{Compliance with ethical standards}

Conflict of interest Helene Ernstberger, Philipp Pieroh, Andreas Höch, Christoph Josten, Steven C. Herath and Georg Osterhoff declare that they have no conflict of interest.

Ethical statement This study was approved by the local institutional ethics committee (Ethik-Kommission Leipzig, 119/19-ek).

Informed consent Not applicable.

Open Access This article is licensed under a Creative Commons Attribution 4.0 International License, which permits use, sharing, adaptation, distribution and reproduction in any medium or format, as long as you give appropriate credit to the original author(s) and the source, provide a link to the Creative Commons licence, and indicate if changes were made. The images or other third party material in this article are included in the article's Creative Commons licence, unless indicated otherwise in a credit line to the material. If material is not included in the article's Creative Commons licence and your intended use is not permitted by statutory regulation or exceeds the permitted use, you will need to obtain permission directly from the copyright holder. To view a copy of this licence, visit http://creativecommons.org/licenses/by/4.0/.

\section{References}

1. Ferguson TA, Patel R, Bhandari M, et al. Fractures of the acetabulum in patients aged 60 years and older: an epidemiological and radiological study. J Bone Jt Surg Br. 2010;92:250-7. 
2. Mears DC. Surgical treatment of acetabular fractures in elderly patients with osteoporotic bone. J Am Acad Orthop Surg. 1999;7:128-41.

3. Letournel É, Judet R, Elson R. Fractures of the acetabulum. Berlin: Springer; 1981.

4. Clarke-Jenssen J, Wikerøy AKB, Røise O, et al. Long-term survival of the native hip after a minimally displaced, nonoperatively treated acetabular fracture. J Bone Jt Surg Am. 2016;98:1392-9.

5. Ryan SP, Manson TT, Sciadini MF, et al. Functional outcomes of elderly patients with nonoperatively treated acetabular fractures that meet operative criteria. J Orthop Trauma. 2017;31:644-9.

6. Harper CM, Lyles YM. Physiology and complications of bed rest. J Am Geriatr Soc. 1988;36:1047-54.

7. Jain R, Basinski A, Kreder HJ. Nonoperative treatment of hip fractures. Int Orthop. 2003;27:11-7.

8. Briffa N, Pearce R, Hill AM, et al. Outcomes of acetabular fracture fixation with ten years' follow-up. J Bone Jt Surg Br. 2011;93:229-36.

9. Cahueque M, Martínez M, Cobar A, et al. Early reduction of acetabular fractures decreases the risk of post-traumatic hip osteoarthritis? J Clin Orthop Trauma. 2017;8:320-6.

10. Anglen JO, Burd TA, Hendricks KJ, et al. The "Gull Sign": a harbinger of failure for internal fixation of geriatric acetabular fractures. J Orthop Trauma. 2003;17:625-34.

11. Carroll EA, Huber FG, Goldman AT, et al. Treatment of acetabular fractures in an older population. J Orthop Trauma. 2010;24:637-44.

12. Borg T, Berg P, Larsson S. Quality of life after operative fixation of displaced acetabular fractures. J Orthop Trauma. 2012;26:445-50.

13. Matta JM. Fractures of the acetabulum: accuracy of reduction and clinical results in patients managed operatively within three weeks after the injury. J Bone Jt Surg Am. 1996;78:1632-45.

14. Gary JL, Paryavi E, Gibbons SD, et al. Effect of surgical treatment on mortality after acetabular fracture in the elderly: a multicenter study of 454 patients. J Orthop Trauma. 2015;29:202-8.

15. Daurka JS, Pastides PS, Lewis A, et al. Acetabular fractures in patients aged 55 years: a systematic review of the literature. Bone Jt J. 2014;96:157-63.

16. Gary JL, VanHal M, Gibbons SD, et al. Functional outcomes in elderly patients with acetabular fractures treated with minimally invasive reduction and percutaneous fixation. J Orthop Trauma. 2012;26:278-83.

17. Mouhsine E, Garofalo R, Borens O, et al. Percutaneous retrograde screwing for stabilisation of acetabular fractures. Injury. 2005;36:1330-6.

18. Pennal GF, Tile M, Waddell JP, et al. Pelvic disruption: assessment and classification. Clin Orthop Relat Res. 1980;151:12-21.

19. Tile M. Pelvic ring fractures: should they be fixed? J Bone Jt Surg Br. 1988;70:1-12.

20. Herath SC, Pott H, Rollmann MFR, et al. Geriatric acetabular surgery: Letournel's contraindications then and now-data from the german pelvic registry. J Orthop Trauma. 2019;33(Suppl 2):S8-S13.

21. Ochs BG, Marintschev I, Hoyer H, et al. Changes in the treatment of acetabular fractures over 15 years: analysis of 1266 cases treated by the German Pelvic Multicentre Study Group (DAO/ DGU). Injury. 2010;41:839-51.

22. Giannoudis PV, Grotz MRW, Papakostidis C, et al. Operative treatment of displaced fractures of the acetabulum: a meta-analysis. J Bone Jt Surg Br. 2005;87:2-9.

23. Sen RK, Veerappa LA. Long-term outcome of conservatively managed displaced acetabular fractures. J Trauma. 2009;67:155-9.

24. Matta JM, Anderson LM, Epstein HC, et al. Fractures of the acetabulum. A retrospective analysis. Clin Orthop Relat Res. 1986;205:230-40.

25. Verbeek DO, van der List JP, Tissue CM, et al. Predictors for long-term hip survivorship following acetabular fracture surgery: importance of gap compared with step displacement. J Bone Jt Surg Am. 2018;100:922-9.

26. Starr AJ, Jones AL, Reinert CM, et al. Preliminary results and complications following limited open reduction and percutaneous screw fixation of displaced fractures of the actabulum. Injury. 2001;32:45-50.

27. Parker PJ, Copeland C. Percutaneous fluoroscopic screw fixation of acetabular fractures. Injury. 1997;28:597-600.

28. Jeffcoat DM, Carroll EA, Huber FG, et al. Operative treatment of acetabular fractures in an older population through a limited ilioinguinal approach. J Orthop Trauma. 2012;26:284-9.

29. Ruchholtz S, Buecking B, Delschen A, et al. The two-incision, minimally invasive approach in the treatment of acetabular fractures. J Orthop Trauma. 2013;27:248-55.

30. Schwabe P, Altintas B, Schaser K-D, et al. Three-dimensional fluoroscopy-navigated percutaneous screw fixation of acetabular fractures. J Orthop Trauma. 2014;28:700-6 (discussion 706).

31. Meena UK, Tripathy SK, Sen RK, et al. Predictors of postoperative outcome for acetabular fractures. Orthop Traumatol Surg Res. 2013;99:929-35.

32. Crowl AC, Kahler DM. Closed reduction and percutaneous fixation of anterior column acetabular fractures. Comput Aided Surg. 2002;7:169-78.

33. Eagle KA, Berger PB, Calkins $\mathrm{H}$, et al. ACC/AHA guideline update for perioperative cardiovascular evaluation for noncardiac surgery-Executive summary. A report of the American College of Cardiology/American Heart Association Task Force on Practice Guidelines (Committee to Update the 1996 Guidelines on Perioperative Cardiovascular Evaluation for Noncardiac Surgery). Anesth Analg. 2002;94:1052-64.

34. Kim JYS, Khavanin N, Rambachan A, et al. Surgical duration and risk of venous thromboembolism. JAMA Surg. 2015;150:110-7.

35. Dock W. The evil sequelae of complete bed rest. JAMA. 1944; 125:1083.

36. Coello R, Glynn JR, Gaspar C, et al. Risk factors for developing clinical infection with methicillin-resistant Staphylococcus aureus (MRSA) amongst hospital patients initially only colonized with MRSA. J Hosp Infect. 1997;37:39-46.

37. Heeg M, Oostvogel HJ, Klasen HJ. Conservative treatment of acetabular fractures: the role of the weight-bearing dome and anatomic reduction in the ultimate results. J Trauma. 1987;27:555-9.

38. Gary JL, Lefaivre KA, Gerold F, et al. Survivorship of the native hip joint after percutaneous repair of acetabular fractures in the elderly. Injury. 2011;42:1144-51. 